\title{
Progression in acute ischemic stroke: Is widespread atherosclerotic background a risk factor?
}

\author{
Mehmet Murat Sümer, ${ }^{1}$ Akçay Övünç Özön ${ }^{2}$ \\ ${ }^{1}$ Department of Neurology, Ankara Güven Hospital, Ankara, Turkey \\ ${ }^{2}$ Department of Neurology, Ankara Liv Hospital, Ankara, Turkey \\ Received: October 19, 2016 Accepted: February 23, 2017 Published online: August 11, 2017
}

\begin{abstract}
Objectives: In this study, we aimed to investigate the causes and conditions related with progression and outcome of progressive acute ischemic stroke.

Patients and methods: In this prospective study, a total of 78 acute ischemic stroke patients (32 males, 46 females; mean age $70 \pm 12.8$ years; range 34 to 95 years) were included between February 2006 and October 2010. The patients were classified into two groups as those with and without progression according to the National Institute of Health Stroke Scale (NIHSS). Risk factors for ischemic stroke, stroke subtypes, and radiological investigations and prognosis were compared between the progressive and non-progressive patients.

Results: Neurological deterioration occurred in 12 patients (15\%). Progressive acute ischemic stroke patients had carotid stenosis compared to non-progressive patients ( $50 \%$ vs $19 \%$ ), and ischemic cardiac disease (33\% vs $6 \%$ ) more common in the patients with progression. Mortality during hospital stay and long term-outcomes were similar between the groups.

Conclusion: Our study results suggest that widespread atherosclerotic diseases may induce neurological progression.
\end{abstract}

Keywords: Acute ischemic stroke; neurological progression; prognosis.

Progression of neurological deficits in ischemic cerebrovascular disease is a poor prognostic factor. Worsening of acute stroke early in its course (within 48 to $72 \mathrm{~h}$ of its onset) is a common occurrence and has potentially serious short- and long-term consequences for the patient. Progression increases mortality, and survivors have more permanent neurological deficits and disabilities ${ }^{[1]}$ The underlying mechanisms are mostly neurological as opposed to the worsening in the later term, and systemic factors such as infection, electrolyte disturbances, myocardial ischemia, and venous thromboembolism tend to play a key role. Systemic atherosclerosis may also affect the cerebrovascular disease; however, there is still no certainty about the causes of progression and prediction of progression. ${ }^{[2]}$

In this study, we aimed to investigate the frequency, risk factors, prognosis, and outcomes of ischemic cerebrovascular disease progression.

\section{PATIENTS AND METHODS}

This prospective study included a total of 78 patients with acute ischemic stroke who were consecutively admitted to Ankara MESA/TOBB hospital between February 2006 and October 2010. All patients who were investigated and treated were hospitalized. Patients who were admitted after more than $24 \mathrm{~h}$ or whose symptoms were completely resolved within $24 \mathrm{~h}$ were excluded. Two patients treated with thrombolytics were also excluded to ensure the homogeneity of the group.

A written informed consent was obtained from each patient or a legal representative. The study protocol was approved by the institutional Ethics Committee. The study was conducted in accordance with the principles of the Declaration of Helsinki.

All patients underwent non-enhanced cranial computed tomography (CT) or magnetic resonance 
imaging (MRI) during the acute phase. Imaging studies were repeated to obtain the exact infarct size and localization during the course of the disease. All patients were examined on a daily basis by a single neurologist during hospitalization. The National Institute of Health Stroke Scale (NIHSS) ${ }^{[3]}$ was applied to all patients upon admission and used on a daily basis during hospitalization. Clinical deterioration was defined as a decline of $\geq 2$ points in the NIHSS ${ }^{[4]}$ If neurological worsening occurred out of the working hours, clinical nurses experienced in assessing NIHSS diagnosed the condition and alerted the clinical physician. In case of a neurological worsening, to identify the possible etiologies, a diagnostic work-up including CT or MRI, blood pressure monitoring, assessment of fluid status, blood viscosity, blood glucose (particularly in patients with diabetes mellitus), arrhythmias, oxygen saturation, and evidence of infection was performed.

The patients were classified into two groups: progressive cerebral infarction (progressive patients; P) and stable cerebral infarction (non-progressive patients; S). The patients were classified as having progressive cerebral infarction, if neurological deterioration was as $\geq 2$ points of increase in the NIHSS. The patients whose symptoms remained stable or improved or whose deterioration was $\leq 2$ points in the NIHSS were classified as having stable cerebral infarction. We avoided recording mild, non-significant fluctuations which were occasionally seen in acute stroke patients, since. The follow-up was continued for six months after hospital discharge. The Rankin scale (RS), ${ }^{[5]}$ which is a well-established widespread and practical scale, was used for surviving patients at three to 28 months (mean: 8 months) to determine functional capacity. A good outcome was defined as having a RS of 0 to 2, while a poor outcome was defined as having a RS of 3 to 5 .

The Trial of Org 10172 in Acute Stroke Treatment $(\text { TOAST })^{[6]}$ classification was used to classify the mechanism of infarction as follows: (i) large artery atherosclerosis, (ii) cardioembolism, (iii) small artery occlusion (lacuna), (iv) stroke of other determined etiology, and $(v)$ stroke of undetermined etiology.

The $\mathrm{P}$ and $\mathrm{S}$ patients were compared for the following variables: demographic characteristics (age, sex, NIHSS on admission), risk factors (hypertension; history of hypertension, use of anti-hypertensive drugs, or consistent measurement of $>160 / 90 \mathrm{mmHg}$ during hospitalization, diabetes mellitus; use of insulin or oral anti-diabetic drugs, fasting blood glucose of $\geq 126 \mathrm{mg} / \mathrm{dL}$, or random blood glucose of $\geq 200 \mathrm{mg} / \mathrm{dL}$, smoking habits including current or previous cigarette smoking during the past five years, ischemic heart disease, previous transient ischemic attack (TIA) or stroke, laboratory data including homocysteine, hemoglobin, fasting glucose, high-density, low-density and very low-density lipoprotein levels, vitamin B12 levels, radiological data including infarct topography, CT-MRI infarct volumes and carotid stenosis, and prognosis including hospital mortality and NIHSS on admission and discharge. The RS was assessed at three to 24 months (mean: 8 months) after discharge and was divided into two categories: 0, 1, 2 points indicate Good outcome which means independence in daily activities and 3, 4, 5 points indicate dependence in daily activities.

Chest X-rays, 12 lead electrocardiographic (ECG) findings, and standard blood and coagulation tests were performed in all patients. Specific blood tests were, however, performed only in selected patients. Chemical tests for blood lipids, glucose, and homocysteine and vitamin B12 levels were performed during the second day of hospitalization under fasting conditions. The patients underwent cardiac and large artery investigations as follows: transthoracic echocardiography was performed in all patients, transesophageal echocardiography in four patients (5.1\%), Doppler carotid ultrasonography in 71 patients (91\%), three-dimensional MRI angiography (extracranial and intracranial) in 20 patients (25\%), and digital subtraction angiography in three patients (3.8\%).

Cranial CT was repeated in all patients after two to three days of neurological stabilization to confirm the localization and to measure the size of the infarct. Cranial MRI was carried out, when the responsible lesion was unable to be visualized or when its size was unable to be properly assessed on CT. The infarct volume (hypodensity zone responsible for the clinical picture) and infarct topography (anteroposterior circulation) were assessed. In total, 42 patients (53.8\%) were assessed with CT and 36 patients (46.2\%) were assessed with MRI. The infarct volume was calculated using the formula $0.5 \times \mathrm{a} \mathrm{b} \times \mathrm{x}$ with $\mathrm{a}, \mathrm{b}$ being the largest perpendicular diameters measured on CT or MRI, and c slice thickness. ${ }^{[7]}$ Cerebral tomography was performed with Hitachi- 4 slice CT scanner and MRI was performed with Siemens - Symphony $1.5 \mathrm{t}$.

The patients were treated for associated illnesses. Treatments for hyperglycemia, hypoxemia, hyperthermia, and low blood pressure were given in accordance with the general directions. High blood pressure was treated with directions of the International Hypertension Treatment Guidelines for 
Acute Ischemic Stroke Patients. In those patients where intracranial hypertension was suspected, intravenous $20 \%$ mannitol was administered. Lowmolecular weight heparins were given to all patients to prevent deep venous thrombosis and pulmonary thromboembolism, except those patients taking unfractionated heparin. All of the P patients were immediately treated with heparin infusion with an activated partial thromboplastin time (aPTT) of two to three times of pretreatment levels, when progression was diagnosed. Warfarin was, then, added in 10 of $12 \mathrm{P}$ patients (83\%), and they were treated for not less than six months with an international normalized ratio (INR) of 1.5 to 2.5 . Anticoagulant treatment was given in 23 of $66 \mathrm{~S}$ patients $(23 \%)$.

\section{Statistical analysis}

The SPSS for Windows version 10 (SPSS Inc., Chicago, IL, USA) was used for the statistical analysis. The Fisher's test was used for noncontinuous data. The Mann-Whitney rank sum test was used in the univariate analysis for continuous data, as the numbers of groups were not equal and/or normally distributed. A $p$ value of $<0.05$ was considered statistically significant.

\section{RESULTS}

Of 78 patients, 32 were men and 46 were women with a mean age of $70 \pm 12.8$ (range, 34 to 95) years. Seventeen patients had large artery atherosclerosis, 22 patients had cardioembolism, 15 patients had small artery occlusion (lacunae), five patients had stroke of other demonstrated etiology, and 19 had stroke of undetermined etiology. In 51 patients, infarcts were located in the anterior circulation, while in 27 patients, they were located in the posterior circulation. Neurological worsening was observed in 12 patients (15.4\%), including seven men and five women with a mean age of $68.3 \pm 11.2$ years. All of the deteriorations occurred within $48 \mathrm{~h}, 10$ were within $24 \mathrm{~h}$, and the other two within the second day of admission. The deterioration affected the level of consciousness in two patients (16.7\%) (one had and additional impaired limb strength and the other had an impaired limb strength and speech). Ten patients (83.3\%) had limb strength (three had an additional speech impairment).

Table 1 shows the demographic data, previous illnesses, risk factors for stroke, and laboratory parameters. There were no statistically significant differences between $\mathrm{P}$ and $\mathrm{S}$ groups in terms of age, sex,

Table 1. Demographic data, risk factors, laboratory parameters, National Institute of Health Stroke Scale, stroke subtypes

\begin{tabular}{|c|c|c|c|c|c|c|c|c|c|}
\hline & \multicolumn{4}{|c|}{ Neurological progression } & \multicolumn{4}{|c|}{ No progression } & \multirow[b]{2}{*}{$p$} \\
\hline & $\mathrm{n}$ & $\%$ & Median & Min-Max & $\mathrm{n}$ & $\%$ & Median & Min-Max & \\
\hline Number of patients & 12 & 15 & & & 66 & 85 & & & \\
\hline \multicolumn{10}{|l|}{ Sex } \\
\hline Male & 7 & 58 & & & 25 & 38 & & & 0.081 \\
\hline Age (year) & & & 70 & $51-84$ & & & 75 & $34-95$ & 0.226 \\
\hline \multicolumn{10}{|l|}{ Risk factors } \\
\hline Hypertension & 8 & 67 & & & 52 & 67 & & & 0.58 \\
\hline Diabetes mellitus & 3 & 25 & & & 16 & 24 & & & 0.700 \\
\hline Ischemic heart disease & 4 & 33 & & & 4 & 6 & & & 0.014 \\
\hline Previous TIA/stroke & 1 & 8 & & & 12 & 18 & & & 0.471 \\
\hline Smoking & 3 & 25 & & & 11 & 17 & & & 0.215 \\
\hline \multicolumn{10}{|l|}{ Laboratory parameters } \\
\hline HDL (mg/dL) & 11 & & 40.5 & $15-81$ & 63 & & 41 & $14-93$ & 0.727 \\
\hline $\mathrm{LDL}(\mathrm{mg} / \mathrm{dL})$ & 12 & & 109 & $41-183$ & 63 & & 108.5 & $45-291$ & 0.162 \\
\hline $\operatorname{VLDL}(\mathrm{mg} / \mathrm{dL})$ & 11 & & 27.5 & $13-49$ & 60 & & 26 & $2-101$ & 0.225 \\
\hline Glucose (mg/dL) & 12 & & 128 & $78-344$ & 66 & & 129 & $86-289$ & 0.517 \\
\hline Hemoglobin (g/dL) & 12 & & 14 & $12.4-15.9$ & 66 & & 13.9 & $8.6-19$ & 0.122 \\
\hline Cyanocobalamine (pg/mL) & 10 & & 363 & $12.8-1684$ & 60 & & 287 & $60-2000$ & 0.353 \\
\hline Homocysteine (mmol/L) & 11 & & 8.55 & $4.7-17.2$ & 55 & & 10.38 & $5.4-26.1$ & 0.067 \\
\hline \multicolumn{10}{|l|}{ Stroke subtype ${ }^{*}$} \\
\hline Large artery atherosclerosis & 5 & 42 & & & 12 & 18 & & & 0.071 \\
\hline Cardioembolism & 2 & 17 & & & 20 & 30 & & & 0.145 \\
\hline Small artery occlusion & 3 & 25 & & & 12 & 18 & & & 0.450 \\
\hline Stroke of other determined etiology & 1 & 8 & & & 4 & 6 & & & 0.827 \\
\hline Stroke of undetermined etiology & 1 & 8 & & & 18 & 27 & & & 0.834 \\
\hline
\end{tabular}

Min: Minimum; Max: Maximum; TIA: Transient ischemic attack; HDL: High density lipoprotein; LDL: Low density lipoprotein; VLDL: Very low-density lipoprotein; ${ }^{\text {p compared }}$ to other four categories combined. 
Table 2. Neuroradiological findings, National Institute of Health Stroke Scale's on admission and at the time of discharge, Rankin scales during follow-up and mortality rates

\begin{tabular}{|c|c|c|c|c|c|c|c|c|c|}
\hline & & Neur & gical prog & ession & & & progressi & & \\
\hline & $\mathrm{n}$ & $\%$ & Median & Min-Max & $\mathrm{n}$ & $\%$ & Median & Min-Max & $p$ \\
\hline Topography & & & & & & & & & 0.542 \\
\hline Anterior circulation & 8 & 67 & & & 43 & 65 & & & \\
\hline Posterior circulation & 4 & 33 & & & 23 & 35 & & & \\
\hline Infarct volume $\left(\mathrm{cm}^{3}\right)$ & & & 248 & $1.35-7891$ & & & 135 & $1.75-12000$ & 0.160 \\
\hline With computed tomography & & & 290 & & & & 130 & & \\
\hline With magnetic resonance imaging & & & 270 & & & & 344 & & \\
\hline Carotid stenosis & & & & & & & & & \\
\hline (occlusion or stenosis $>70 \%$ ) & 6 & 50 & & & 12 & 19 & & & 0.035 \\
\hline NIHSS on admission & 12 & & 8.5 & $0-22$ & 66 & & 4 & $0-24$ & 0.322 \\
\hline NIHSS on discharge & 12 & & 5.5 & $0-19$ & 58 & & 2 & $0-22$ & 0.021 \\
\hline Rankin $0-2$ & 12 & & 7 & 58 & 47 & & 36 & 77 & 0.178 \\
\hline Mortality & 1 & 8 & & & 5 & 7 & & & 0.160 \\
\hline
\end{tabular}

Min: Minimum; Max: Maximum; NIHSS: National Institute of Health Stroke Scale.

hypertension, diabetes mellitus, previous TIA/stroke, smoking habits, lipid levels, and glucose levels upon admission, hemoglobin, vitamin B12 levels, and stroke subtypes. Patients with progressive cerebrovascular diseases had more frequent previous heart disease, compared to stable cerebrovascular diseases $(\mathrm{p}=0.01)$. Stroke subtypes were not different between $\mathrm{P}$ and $\mathrm{S}$ patients. However, a higher number of $\mathrm{P}$ patients $(6 / 12$; $50 \%$ ) had carotid occlusion or severe stenosis (more than $70 \%)$, compared to S patients $(12 / 66 ; 19 \%)$, indicating a statistically significant difference $(\mathrm{p}=0.03)$.

In the patients with neurological progression, the responsible infarcts were assessed with CT in seven and with MRI in five of 12 patients. The lesions were assessed with CT in 21 and with MRI in 45 of 66 patients in the non-progressive infarct group. For the patients with neurological progression, CT and MRI were repeated after the progression ended, and neuroradiological findings with and without progression were compared (Table 2). As for the lesion topography, anterior or posterior location of the infarcts was not different in $\mathrm{P}$ or $\mathrm{S}$ patients. However, the mean infarct volumes were higher in the progressive infarct subtypes as assessed by CT and with MRI, compared to the stable group. However, it did not reach statistical significance.

The NIHSS scores on admission and at the time of discharge and functional status as assessed by the RS (0-2: independent in daily activities; $3-5$ : dependent on daily activities) on the follow-up examination were compared (Table 2). The NIHSS scores on admission were found to be higher in the patients with progressive disease, compared to those with stable cerebral vascular lesions; however, it did not reach statistical significance. On the other hand, the patients with progressive cerebrovascular diseases had worse NIHSS scores, compared to those with stable disease patients at the time of discharge (Table 2). The mortality rates during hospitalization were not statistically different between the $\mathrm{P}$ and $\mathrm{S}$ patients.

In addition, the $\mathrm{P}$ patients had a longer median hospital stay (7.5; range, 3 to 28 days), compared to the $\mathrm{S}$ patients (5; range, 2 to 30 days) $(\mathrm{p}=0.04)$. Although the patients with stable disease were more independent during the follow-up examination, compared to the patients with progressive disease ( $77 \%$ vs $58 \%)$, the difference was not statistically significant.

\section{DISCUSSION}

In the present study, the patients with progressive cerebrovascular disease more frequently had previous ischemic heart diseases and more frequently had severe carotid stenosis than stable ischemic cerebrovascular disease patients.

The incidence of neurological deterioration has been reported with a wide range of variations for the definition of neurological deterioration, and this concerns the time frame (early: within first $24 \mathrm{~h}$ or within $72 \mathrm{~h}$ ), neurological stroke scale used (i.e., NIHSS vs Canadian Stroke Scale), progression level (NIHSS 2 or 5 points), and the treatment used for these patients (non-thrombolyzed - thrombolyzed). ${ }^{[8,9]}$ The frequency of neurological deterioration in this singlecenter study was $15.4 \%$, which was higher than that of a retrospective study which assessed deteriorations within the first two days ${ }^{[10]}$ and lower than those of two prospective studies (35\% and $37 \%$, respectively). ${ }^{[1,12]}$ The studies for early neurological deterioration in non- 
thrombolyzed patients were found to have frequencies between 13 to $37 \%^{[13-17]}$ Our patients with neurological progression had worse NIHSS scores on discharge, compared to non-progressive patients ( 8 vs 3.7 ), while the NIHSS scores were not different on admission between these two groups. These findings are consistent with previous reports. ${ }^{[12]}$ However, the in-hospital mortality rates were not different between the groups, as different from the study of Siegler et al. ${ }^{[1]}$

Dávalos et al. ${ }^{[15]}$ reported that coronary artery diseases were more frequent in progressing ischemic stroke patients. Carotid stenosis is a well-known risk factor for ischemic cerebrovascular disease. In this study, we also found that the patients with progressing deficits had more frequently severe carotid stenosis than the patients with stable deficits. Proximal arterial occlusion was also observed more frequently in the patients with neurological deterioration in previous studies. ${ }^{[2,13,16,18]}$ This is particularly important on ischemic penumbra in which the level of the decline in the vascular supply was correlated with the ondulations of clinical symptoms. This finding is also of utmost importance for the management of acute ischemic stroke patients, particularly with carotid stenosis. The avoidance of lowering blood pressure to critical levels which may lead the ischemic penumbra to infarction in acute ischemic stroke patients is the mainstay of modern widespread management of acute stroke treatment. Proximal occlusions entail larger volumes of hypoperfused tissues. ${ }^{[19]}$ This increases the risk of further extension of penumbral tissue volumes and levels and, hence, the infarction. Our finding which emphasizes increasing cerebral perfusion pressures for salvation of ischemic penumbra is particularly important for patients with severe carotid stenoses whose neurological deficits are progressing.

These two findings also indicate that progressing ischemic stroke patients are more likely to have widespread atherosclerotic disease than stable patients. In another words, patients with widespread atherosclerotic disease background may develop cerebrovascular disease, and this would more probably be progressing type. A contributing role of poor collateral blood supply is suggested by the fact that coronary artery disease is often associated with a higher prevalence of severe extracranial or intracranial atherosclerotic disease. In a study with acute subcortical infarcts, progressive neurological deficits were found more frequently in patients having arterial stiffness. ${ }^{[20]}$ Arterial stiffness is a marker of vascular endothelial impairment and arteriosclerosis. ${ }^{[1,21]}$
Our results showed that the patients with ischemic heart disease or carotid stenosis had more frequently progressing neurological deficits than those without these diseases. This finding suggests that patients with progressing neurological deficits may have a chronic atherosclerotic background.

The risk of death or dependency at three months of the patients with neurological progression were higher than stable stroke patients in most studies. ${ }^{[2,15,22-24]}$ In our study, the mortality rates on discharge were not different between groups. Although the patients with progressive disease had higher NIHSS scores on admission, it did not reach statistical significance. On the other hand, these patients had significantly higher NIHSS scores on discharge. Independent patients were also similar between the groups during follow-up in our study in contrast to the other studies. These findings also confirm our previous results on the same issue. ${ }^{[25]}$

Functional outcome at sixth month were not different in progressive and non-progressive stroke patients; the rates of independent patients were nearly the same. This finding indicates that, although these are neurologically more handicapped patients, compared to stable stroke patients, they can show better gradual improvement at hospital discharge.

Nonetheless, this study has certain shortcomings. First, our sample size relatively small which prevents us from reaching definite conclusions. Second, as this is a clinical study, we can only compare the groups with associated conditions and diseases. Pathophysiological directions are beyond the scope of this study. However, the controversial findings on progression in ischemic stroke should urge us to conduct more studies on general mechanisms which should include investigations on detailed mechanisms and treatment options by including larger numbers of patients.

In conclusion, our study suggest that widespread atherosclerotic diseases may trigger neurological progression.

\section{Declaration of conflicting interests}

The authors declared no conflicts of interest with respect to the authorship and/or publication of this article.

\section{Funding}

The authors received no financial support for the research and/or authorship of this article. 


\section{REFERENCES}

1. Kim J, Cha MJ, Lee DH, Lee HS, Nam CM, Nam HS, et al. The association between cerebral atherosclerosis and arterial stiffness in acute ischemic stroke. Atherosclerosis 2011;219:887-91.

2. Mori M, Naganuma M, Okada Y, Hasegawa Y, Shiokawa Y, Nakagawara J, et al. Early neurological deterioration within 24 hours after intravenous rt-PA therapy for stroke patients: the Stroke Acute Management with Urgent Risk Factor Assessment and Improvement rt-PA Registry. Cerebrovasc Dis 2012;34:140-6.

3. "Tissue plasminogen activator for acute ischemic stroke. The National Institute of Neurological Disorders and Stroke rt-PA Stroke Study Group." N Engl J Med 1995;333:1581-7.

4. Castillo J. Deteriorating stroke: diagnostic criteria, predictors, mechanisms and treatment. Cerebrovasc Dis 1999;9:1-8.

5. Rankin J. Cerebral vascular accidents in patients over the age of 60. II. Prognosis. Scott Med J 1957;2:200-15.

6. Adams HP Jr, Bendixen BH, Kappelle LJ, Biller J, Love $\mathrm{BB}$, Gordon DL, et al. Classification of subtype of acute ischemic stroke. Definitions for use in a multicenter clinical trial. TOAST. Trial of Org 10172 in Acute Stroke Treatment. Stroke 1993;24:35-41.

7. Dávalos A, Castillo J, Marrugat J, Fernandez-Real JM, Armengou A, Cacabelos P, et al. Body iron stores and early neurologic deterioration in acute cerebral infarction. Neurology 2000;54:1568-74.

8. Seners P, Turc G, Oppenheim C, Baron JC. Incidence, causes and predictors of neurological deterioration occurring within $24 \mathrm{~h}$ following acute ischaemic stroke: a systematic review with pathophysiological implications. J Neurol Neurosurg Psychiatry 2015;86:87-94.

9. Simonsen CZ, Schmitz ML, Madsen MH, Mikkelsen IK, Chandra RV, Leslie-Mazwi T, et al. Early neurological deterioration after thrombolysis: Clinical and imaging predictors. Int J Stroke 2016;11:776-82.

10. Maier IL, Bauerle M, Kermer P, Helms HJ, Buettner T. Risk prediction of very early recurrence, death and progression after acute ischaemic stroke. Eur J Neurol 2013;20:599-604.

11. Siegler JE, Boehme AK, Kumar AD, Gillette MA, Albright KC, Martin-Schild S. What change in the National Institutes of Health Stroke Scale should define neurologic deterioration in acute ischemic stroke? J Stroke Cerebrovasc Dis 2013;22:675-82.

12. Siegler JE, Boehme AK, Albright KC, George AJ, Monlezun DJ, Beasley TM, et al. A proposal for the classification of etiologies of neurologic deterioration after acute ischemic stroke. J Stroke Cerebrovasc Dis 2013;22:549-56.
13. Dávalos A, Cendra E, Teruel J, Martinez M, Genís D. Deteriorating ischemic stroke: risk factors and prognosis. Neurology 1990;40:1865-9.

14. Toni D, Fiorelli M, Gentile M, Bastianello S, Sacchetti $\mathrm{ML}$, Argentino C, et al. Progressing neurological deficit secondary to acute ischemic stroke. A study on predictability, pathogenesis, and prognosis. Arch Neurol 1995;52:670-5.

15. Dávalos A, Toni D, Iweins F, Lesaffre E, Bastianello S, Castillo J. Neurological deterioration in acute ischemic stroke: potential predictors and associated factors in the European cooperative acute stroke study (ECASS) I. Stroke 1999;30:2631-6.

16. Alexandrov AV, Felberg RA, Demchuk AM, Christou I, Burgin WS, Malkoff $M$, et al. Deterioration following spontaneous improvement: sonographic findings in patients with acutely resolving symptoms of cerebral ischemia. Stroke 2000;31:915-9.

17. Grotta JC, Welch KM, Fagan SC, Lu M, Frankel MR, Brott $\mathrm{T}$, et al. Clinical deterioration following improvement in the NINDS rt-PA Stroke Trial. Stroke 2001;32:661-8.

18. Rubiera M, Ribo M, Delgado-Mederos R, Santamarina E, Delgado P, Montaner J, et al. Tandem internal carotid artery/middle cerebral artery occlusion: an independent predictor of poor outcome after systemic thrombolysis. Stroke 2006;37:2301-5.

19. Copen WA, Rezai Gharai L, Barak ER, Schwamm LH, Wu $\mathrm{O}$, Kamalian S, et al. Existence of the diffusion-perfusion mismatch within 24 hours after onset of acute stroke: dependence on proximal arterial occlusion. Radiology 2009;250:878-86.

20. Saji N, Kimura K, Kawarai T, Shimizu H, Kita Y. Arterial stiffness and progressive neurological deficit in patients with acute deep subcortical infarction. Stroke 2012;43:3088-90.

21. Pantoni L. Cerebral small vessel disease: from pathogenesis and clinical characteristics to therapeutic challenges. Lancet Neurol 2010;9:689-701.

22. Dharmasaroja PA, Muengtaweepongsa S, Dharmasaroja P. Early outcome after intravenous thrombolysis in patients with acute ischemic stroke. Neurol India 2011;59:351-4.

23. Siegler JE, Martin-Schild S. Early Neurological Deterioration (END) after stroke: the END depends on the definition. Int J Stroke 2011;6:211-2.

24. Asplund K. Any progress on progressing stroke? Cerebrovasc Dis 1992;2:317-9.

25. Sumer M, Ozdemir I, Erturk O. Progression in acute ischemic stroke: frequency, risk factors and prognosis. J Clin Neurosci 2003;10:177-80. 